\title{
The Utility of FDG-PET for Managing Patients with Malignant Lymphoma: Analysis of Data from a Single Cancer Center
}

\author{
Osamu Imataki ${ }^{1}$, Yotaro Tamai ${ }^{1}$, Koiku Yokoe $^{2}$, \\ Takayoshi Furukawa ${ }^{2}$ and Kimihiro Kawakami ${ }^{1}$
}

\begin{abstract}
Objective ${ }^{18} \mathrm{~F}$-fluorodeoxyglucose (FDG) positron-emission tomography (PET) has been widely applied to malignant lymphoma both for initial staging and response evaluation. The objective is to compare the efficacy of the less common, but more easily implemented modality, CT, with that of FDG.

Methods We retrospectively reviewed consecutive patients diagnosed with malignant lymphoma in our hospital between October 2002 and March 2006, and compared the efficacy of FDG-PET and CT. The standard reference was defined by the pathology and clinical course of patients followed for more than 3 months.

Results Thirty-three cases for staging and 62 cases for response evaluation after treatment were included. We calculated the sensitivity and specificity of each modality. The accuracy of the diagnostic modality was evaluated using receiver operating characteristic (ROC) analysis. The sensitivity and specificity of the initial staging were $87 \%$ and $100 \%$ on CT evaluation and $87 \%$ and $100 \%$ on FDG-PET, respectively. Sensitivity and specificity of the re-staging were $81 \%$ and $78 \%$ on CT evaluation and $82 \%$ and $97 \%$ on FDG-PET, respectively. The diagnostic accuracy of FDG-PET was comparable with that of CT both in initial staging and response evaluation. The maximum standardized uptake value was not associated with patient survival. In subgroup analysis, a tendency of lower sensitivity in the initial staging was found in FDG-PET for follicular lymphoma and CT for diffuse large B-cell lymphoma.

Conclusion Although different staging procedures appear better suited to certain subtypes of lymphoma, in general CT imaging might be as useful as FDG-PET in initial staging in selected patients.
\end{abstract}

Key words: malignant lymphoma, fluorodeoxyglucose positron-emission tomography (FDG-PET), clinical stage

(Inter Med 48: 1509-1513, 2009)

(DOI: 10.2169/internalmedicine.48.1856)

\section{Introduction}

Accurate staging and re-staging is crucial for the optimal management of malignant lymphoma. It is widely known that positron emission tomography (PET) with ${ }^{18} \mathrm{~F}-$ fluorodeoxyglucose (FDG) is useful for the determination of disease stage $(1,2)$ and prediction of the clinical prognosis for malignant lymphoma patients (3-5). Although the sensitivity of evaluation by PET with FDG (FDG-PET) is higher than that of conventional computed tomography (CT) scanning in detecting lymphoma lesions, false positives inevitably occur in some subtypes of lymphoma, decreasing the specificity of FDG-PET (6). It is well known that biologic differences between specific pathologic subtypes of lymphoma result in different degrees of FDG uptake (7).

For lymphoma, FDG-PET shows a high sensitivity but is not specific for malignancy, since FDG is transported into cells requiring glucose as energy by its transporters (8). Most of the misreading of FDG-PET imaging for malignant

${ }^{1}$ Division of Hematology \& Stem Cell Transplantation, Shizuoka Cancer Center, Shizuoka and ${ }^{2}$ Division of Diagnostic Radiology, Shizuoka Cancer Center, Shizuoka

Received for publication November 10, 2008; Accepted for publication May 27, 2009

Correspondence to Dr. Osamu Imataki, oima@med.kagawa-u.ac.jp 
lymphoma involves inflammatory non-tumor diseases (9). Castellucci et al reported the potential pitfalls of FDG-PET imaging for patients with malignant lymphoma, and concluded that an accurate patient history and a skillful nuclear medicine physician are very important factors to avoid misinterpretation when reading FDG-PET scans (10). They also emphasized the usefulness of hybrid FDG-PET/CT in order to identify non-tumoral FDG focal uptakes (10).

Thus, 3 major factors are essential for an accurate diagnosis of malignant lymphoma on FDG-PET imaging. One is the host factor: the subtype of histopathology. The second is the physician factor: the skillfulness of the nuclear medicine physicians. The third is the imaging factor: the anatomic perceptiveness of the imaging technique, i.e. the performance of FDG-PET/CT. In cancer centers, physicians and radiologists are familiar with malignancies and differential diagnoses of malignant and benign diseases. Internal and radiological specialists dealing with oncology in cancer centers may show great sensitivity but little specificity for malignancy. We assessed how the sensitivity and specificity may increase with the single or combined use of FDG-PET and $\mathrm{CT}$ in the clinical evaluation of malignant lymphoma, and also the ability to evaluate the bone marrow involvement of lymphoma cells by FDG-PET in the setting of a single cancer center survey.

\section{Patients and Methods}

\section{Patient population}

We retrospectively reviewed consecutive patients diagnosed as having malignant lymphomas in our hospital between October 2002 and March 2006. Diagnosis was confirmed histologically, and non-malignant disease was excluded. We collected 33 cases of initial staging before treatment and 62 cases of disease evaluation (re-staging) after treatment. All cases received FDG-PET and CT concomitantly, or simultaneously (known as FDG-PET/CT).

\section{FDG-PET/CT imaging and FDG-PET imaging}

FDG-PET/CT imaging was conducted 1 hour after the administration of $180 \mathrm{MBq}$ of FDG (3D mode, 2.5 minutes/ bed position) combined with dual-slice helical CT images (Discovery ST, GE Medical Systems). FDG-PET and CT were also performed independently. FDG-PET imaging was conducted 1 hour after the administration of $230 \mathrm{MBq}$ of FDG (2D mode, 5 minutes/bed position) (Advance $\mathrm{NXi}$, GE); additionally, CT images were used for FDG-PET attenuation correction. The standard reference for the lesion was determined by the overall clinical information including the course and findings in patients whose follow-up periods lasted for more than 3 months after the diagnosis.

\section{Clinical and statistical evaluation}

We calculated the sensitivity and specificity of each modality and combined modality according to the standard ref- erence, as defined above. Radiological diagnosis was made by more than 2 radiologists and findings were reviewed by 2 hematologists. We used Cheson's response criteria for evaluating the response (11). The accuracy of a diagnostic modality was evaluated using receiver operating characteristic (ROC) analysis. We tested the correlation between 2 independent parameters [maximum standardized uptake value (SUVmax) and survival] with the $\mathrm{r}^{2}$ value. Overall survival from the date of diagnosis was estimated for survival analysis. A student t-test was used to determine differences in subset data for subgroups of lymphoma histology. Differences were considered statistically significant at a $P$ value of less than 0.05 .

In patients proven to show bone marrow involvement of lymphoma cells, we evaluated the diagnostic accuracy of FDG-PET imaging. FDG-PET preceded the bone marrow evaluation.

\section{Results}

Lymphoma subtypes in the 95 patients comprised 21 cases of Hodgkin's lymphoma, 28 of indolent lymphoma, 37 of aggressive lymphoma, and 9 of T-cell lymphoma. Indolent lymphoma included 21 follicular lymphomas, 2 mucosa-associated lymphoid tissue (MALT) lymphomas, 2 lymphoplasmacytic lymphomas and 3 splenic marginal zone lymphomas. Aggressive lymphoma included 33 diffuse large B-cell lymphomas, 3 mantle cell lymphomas and 1 intravascular large B-cell lymphoma. The consistency of initial staging (Ann Arbor classification) by CT and FDG-PET was $75.8 \%$ (25/33), with 8 discordant cases including 5 upstaged cases and 3 downstaged cases ( 3 diffuse large B cell, 2 Hodgkin's, 1 follicular and 2 T-cell lymphomas) by FDGPET. On the other hand, consistency of re-staging after chemotherapy by CT and FDG-PET was $74.2 \%$ (46/62), with 16 discordant cases including 5 upstaged cases and 11 downstaged cases (3 diffuse large B cell, 6 Hodgkin's, 5 follicular and 2 T-cell lymphomas) by FDG-PET. In the response evaluation, FDG-PET showed a greater tendency to downstage rather than upstage the disease. Response definitions after initial treatment were 53\% (33/62) in CR, $11 \%$ $(7 / 62)$ in PR, 31\% (19/62) in SD and 5\% (3/62) in PD. In diffuse large B cell lymphoma, they were $73 \%$ (19/26), 8\% $(2 / 26), 19 \%(5 / 26)$ and $0 \%$, respectively. In follicular lymphoma, they were $31 \%(4 / 13), 15 \%(2 / 13), 39 \%$ (5/13) and $15 \%$ (2/13), respectively. In Hodgkin lymphoma, they were $40 \%$ (6/15), 13\% (2/15), 47\% (7/15) and 0\%, respectively.

Compared to the standard reference, each modality had about the same accuracy for staging. Before treatment, FDG-PET facilitated correct upstaging in two of 4 patients in whom CT staging at diagnosis was incorrect (50\%). Conversely, CT facilitated accurate staging in six of 8 patients in whom FDG-PET staging was incorrect (75\%, upstaging in 2 and downstaging in 4 patients). After chemotherapy, FDG-PET facilitated accurate staging in eleven of 13 patients in whom CT staging was incorrect $(85 \%$, upstaging in 
3 and downstaging in 8) and CT facilitated accurate staging in four of 6 patients $(75 \%$, upstaging in 3 and downstaging in 1).

Sensitivity and specificity in initial staging were $87 \%$ and $100 \%$ on CT evaluation and $87 \%$ and $100 \%$ on FDG-PET, respectively. Sensitivity and specificity in re-staging after chemotherapy were $81 \%$ and $78 \%$ on CT evaluation and $82 \%$ and $97 \%$ on FDG-PET, respectively. The ROC curve showed that the accuracy of diagnosis by CT and FDG-PET was comparable in the initial staging before treatment. However, in re-staging after treatment, the accuracy of diagnosis by CT was lower than that by FDG-PET (Fig. 1). A positive predictive value (PPV) and negative predictive value (NPV) were also calculated and are presented in Table 1.

We analyzed the association between overall survival and various parameters. Successful combined use of the 2 modalities (CT and FDG-PET) was not associated with patients' survival $(\mathrm{p}=0.5)$. We did not observe an apparent correlation between SUVmax and survival $\left(r^{2}=0.10\right)$. In sub-

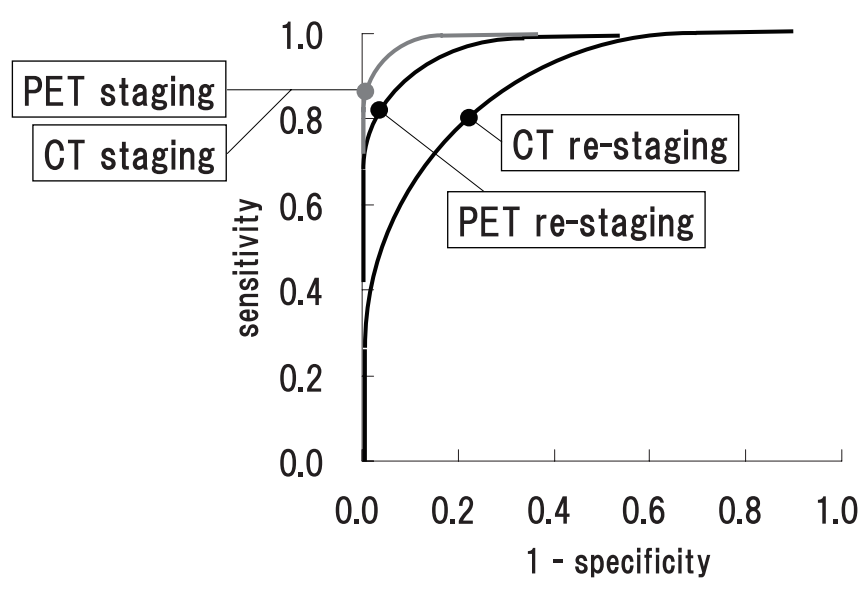

Figure 1. ROC curve of the diagnostic accuracy of CT and FDG-PET. The ROC curve revealed that the accuracy of diagnosis by CT and FDG-PET was comparable for both initial staging before and re-staging after treatment. group analysis, there was no statistical association between SUVmax and lymphoma subtype (Hodgkin's lymphoma, follicular lymphoma and diffuse large B-cell lymphoma). However, a tendency of lower sensitivity in the initial staging was found in FDG-PET for follicular lymphoma and CT for diffuse large B-cell lymphoma. Regarding the diagnosis for Hodgkin's lymphoma, FDG-PET in initial staging and CT in re-staging had relatively low specificity, respectively. Table 2 shows the sensitivity and specificity by subgroup analysis according to subtype of lymphoma histology.

In all 7 patients diagnosed as showing bone marrow infiltration of lymphoma cells by FDG-PET, no histological confirmation of involvement was obtained. Our study patients included 12 lymphoma patients with extranodal lesions (except for bone marrow invasion). In 4 out of these 12 patients, the staging by CT was not in accordance with that by PET. In 2 patients, CT underestimated the stage compared with PET; in another 2 patients PET underestimated the stage compared with CT. Therefore, in our study patients, there was no obvious benefit in evaluating extranodal lesions by PET rather than CT. However, a combined evaluation contributed to avoiding the underestimation in $33 \%(4 / 12)$ of cases having extranodal lesions.

\section{Discussion}

Positron emission tomography (PET) has become a very useful technique for staging and monitoring the therapy response regarding lymphoma, providing unique information on the biological behavior of the disease. Increased ${ }^{18} \mathrm{~F}-\mathrm{FDG}$ uptake by lymphomas is based on elevated glycolysis and a longer residence time of FDG in malignant cells compared with most normal tissues, and this technique was first applied clinically by Paul in 1987 (12). In general, considering the sensitivity and specificity of the examination, a higher sensitivity leads to a lower specificity and vice versa according to a change in the cut-off level in the setting of a single modality. Although computed tomography (CT) is the prin-

Table 1. Sensitivity, Specificity, PPV, and NPV of CT and FDG-PET Modalities before and after Treatment

\begin{tabular}{|c|c|c|c|c|}
\hline & sensitivity & specificity & PPV & NPV \\
\hline \multicolumn{5}{|l|}{ Staging } \\
\hline CT $(n=33)$ & 0.87 & 1.00 & 1.00 & 0.43 \\
\hline FDG-PET $(n=33)$ & 0.87 & 1.00 & 1.00 & 0.43 \\
\hline \multicolumn{5}{|l|}{ Re-staging } \\
\hline $\mathrm{CT}(\mathrm{n}=62)$ & 0.81 & 0.78 & 0.72 & 0.85 \\
\hline FDG-PET $(n=62)$ & 0.82 & 0.97 & 0.96 & 0.87 \\
\hline
\end{tabular}

Abbreviations: PPV: positive predictive value, NPV: negative predictive value 
Table 2. Sensitivity and Specificity of CT and FDG-PET Modality before and after Treatment according to Histology Type

\begin{tabular}{|l|l|l|l|l|l|l|l|}
\hline & \multicolumn{2}{|l|}{ Hodgkin's lymphoma } & \multicolumn{2}{l}{ Follicular lymphoma } & \multicolumn{2}{l|}{$\begin{array}{l}\text { Diffuse large B-cell } \\
\text { lymphoma }\end{array}$} \\
\hline & \multicolumn{2}{|l|l|l|l|l|l|l|l|}{ sensitivity } & specificity & sensitivity & specificity & sensitivity & specificity \\
\hline Staging & $\mathrm{n}=6$ & $\mathrm{n}=8$ & $\mathrm{n}=7$ & \\
\hline CT & 1.00 & 1.00 & 1.00 & 1.00 & 0.71 & 1.00 \\
\hline FDG-PET & 1.00 & 0.67 & 0.88 & 1.00 & 1.00 & 1.00 \\
\hline Re-staging & $\mathrm{n}=15$ & & $\mathrm{n}=13$ & & $\mathrm{n}=26$ & \\
\hline CT & 1.00 & 0.67 & 0.85 & 0.85 & 0.96 & 0.96 \\
\hline FDG-PET & 1.00 & 0.93 & 0.92 & 1.00 & 0.92 & 1.00 \\
\hline
\end{tabular}

cipal imaging modality for the staging and re-staging of lymphoma, numerous reports have found that FDG-PET, which provides metabolic information, is more sensitive than anatomical imaging modalities (13-16). However, since FDG is not specific for malignancy, 3 variables were identified as modulating the ability of FDG-PET to discriminate non-malignant lesions: one is the subtype of lymphoma; the second is the amount of physiological uptake; and the third is the presence or not of an inflammatory lesion. Thus, FDG-PET has played an important role in staging, monitoring the response to therapy, and performing follow-up; however, the sensitivity of FDG-PET depends on the subtype of lymphoma and the extent and location of disease. These issues are essential pitfalls in the determination of lymphoma staging, especially after the modification of chemotherapy or radiation therapy. Therefore, optimal timing seems to be crucial since a transient increase in the stromal reaction may result in overestimation of the fraction of viable cells (17).

In the present study, downstaging by FDG-PET occurred at a similar rate as upstaging in the initial diagnosis. The utility of CT as well as FDG-PET in initial staging was very high on evaluation by the ROC curve before treatment. This is thought to be due to the high frequency of lymphoma lesions undergoing FDG-PET in our cancer center. The radiologists judged the suspected lesions as 'positive' more sensitively using CT imaging, which resulted in the same rates of both sensitivity and specificity for CT as for FDG-PET. So, we concluded that CT exhibited a comparable efficacy for detecting truly positive lesions in the initial staging.

On the other hand, on evaluating treatment efficacy, CT was more specific but FDG-PET was more sensitive, and the diagnostic accuracy was higher for FDG-PET than CT using the ROC curve. This means that FDG-PET tended to exaggerate the accumulation. Indeed, indolent lymphoma including follicular lymphoma, mucosa-associated lymphoid tissue (MALT) lymphoma, and Hodgkin's lymphoma tended to reveal a negative uptake after chemotherapy, so the CT findings were downstaged by FDG-PET. We recognized that the utility after treatment of FDG-PET was superior to that of CT, due to the influence of treatment modification. Nonetheless, CT imaging has significant shortcomings, particularly in the post-therapy setting while low anatomical resolution is a technical limitation of FDG-PET; therefore, the combined use of FDG-PET and CT imaging may overcome these shortcomings (18).

Pakos et al evaluated the usefulness of FDG-PET for diagnosing the involvement of bone marrow with a systematical review, and showed good diagnostic performance with relatively lower sensitivity $(51 \%)$ and higher specificity (91\%). They concluded that FDG-PET may complement a diagnosis by bone marrow biopsy (19). As in the present study, the bone marrow involvement of lymphoma cells could not be accurately detected by FDG-PET.

The diagnostic accuracy of FDG-PET was the same as that of CT in the initial staging in the present study, although the diagnostic performance of FDG-PET was higher than that of CT in re-staging. For initial staging of follicular lymphoma, FDG-PET seemed to be less sensitive than CT, while CT tended to be less sensitive than FDG-PET in diffuse large B-cell lymphoma. In response evaluation, FDGPET was more effective in all kinds of lymphoma. The diagnostic accuracy of the CT modality was decreased after treatment, especially for Hodgkin's lymphoma. As indicated in this result, different lymphoma subtypes are more accurately diagnosed by different modalities. The diagnosis of bone marrow involvement by FDG-PET was also unreliable. Taken together, these results suggest that if selected patients were examined and selected radiologists reviewed images, CT imaging might be of at least equal value compared to FDG-PET in the initial staging. In this situation overdiagnosis is likely to occur in staging using FDG-PET. This circumstance would be characterized not only in our cancer center but also in other cancer centers. Physicians should be aware that the high sensitivity of FDG-PET might lead to over-treatment. 


\section{References}

1. Sasaki M, Kuwabara Y, Koga H, et al. Clinical impact of whole body FDG-PET on the staging and therapeutic decision making for malignant lymphoma. Ann Nucl Med 16: 337-345, 2002.

2. Tatsumi M, Cohade C, Nakamoto Y, et al. Direct comparison of FDG PET and CT findings in patients with lymphoma: initial experience. Radiology 237: 1038-1045, 2005.

3. Hutchings M, Loft A, Hansen M, et al. FDG-PET after two cycles of chemotherapy predicts treatment failure and progression-free survival in Hodgkin's lymphoma. Blood 107: 52-59, 2006.

4. Juweid ME, Wiseman GA, Vose JM, et al. Response assessment of aggressive non-Hodgkin's lymphoma by integrated International Workshop Criteria and fluorine-18-fluorodeoxyglucose positron emission tomography. J Clin Oncol 23: 4652-4661, 2005.

5. Spaepen K, Stroobants S, Dupont P, et al. Prognostic value of positron emission tomography (PET) with fluorine-18 fluorodeoxyglucose ( $\left.\left[{ }^{18} \mathrm{~F}\right] \mathrm{FDG}\right)$ after first-line chemotherapy in nonHodgkin's lymphoma: is $\left[{ }^{18} \mathrm{~F}\right] \mathrm{FDG}-\mathrm{PET}$ a valid alternative to conventional diagnostic methods? J Clin Oncol 19: 414-419, 2001.

6. Bomanji JB, Costa DC, Ell PJ. Clinical role of positron emission tomography in oncology. Lancet Oncol 2: 157-164, 2001.

7. Elstrom R, Guan L, Baker G, et al. Utility of FDG-PET scanning in lymphoma by WHO classification. Blood 101: 3875-3876, 2003.

8. Gallagher BM, Fowler JS, Gutterson NI, et al. Metabolic trapping as a principle of radiopharmaceutical design: some factors responsible for the biodistribution of $\left[{ }^{18} \mathrm{~F}\right]$ 2-deoxy-2-fluoro-D-glucose. J Nucl Med 19: 1154-1161, 1978.

9. Barrington SF, O'Doherty MJ. Limitations of PET for imaging lymphoma. Eur J Nucl Med Mol Imaging 30 Suppl 1 : S117-127, 2003.

10. Castellucci P, Nanni C, Farsad M, et al. Potential pitfalls of ${ }^{18} \mathrm{~F}$ FDG PET in a large series of patients treated for malignant lym- phoma: prevalence and scan interpretation. Nucl Med Commun 26: 689-694, 2005.

11. Cheson BD, Pfistner B, Juweid ME, et al; The International Harmonization Project on Lymphoma. Revised response criteria for malignant lymphoma. J Clin Oncol 25: 579-586, 2007.

12. Paul R. Comparison of fluorine-18-2-fluorodeoxyglucose and gallium-67 citrate imaging for detection of lymphoma. J Nucl Med 28: 288-292, 1987.

13. Moog F, Bangerter M, Diederichs CG, et al. Lymphoma: role of whole-body 2-deoxy-2-[F-18]fluoro-D-glucose (FDG) PET in nodal staging. Radiology 203: 795-800, 1997.

14. Stumpe KD, Urbinelli M, Steinert HC, et al. Whole-body positron emission tomography using fluorodeoxyglucose for staging of lymphoma: effectiveness and comparison with computed tomography. Eur J Nucl Med 25: 721-728, 1998.

15. Schiepers C. PET for staging of Hodgkin's disease and nonHodgkin's lymphoma. Eur J Nucl Med Mol Imaging 30: S82-88, 2003.

16. Spaepen K. Positron emission tomography with $\left[{ }^{18} \mathrm{~F}\right] \mathrm{FDG}$ for therapy response monitoring in lymphoma patients. Eur J Nucl Med Mol Imaging 30: S97-105, 2003.

17. Spaepen K, Stroobants S, Dupont P, et al. [(18)F]FDG PET monitoring of tumour response to chemotherapy: does [(18)F] FDG uptake correlate with the viable tumour cell fraction? Eur $\mathrm{J}$ Nucl Med Mol Imaging 30: 682-688, 2003.

18. Freudenberg LS, Antoch G, Schutt P, et al. FDG-PET/CT in restaging of patients with lymphoma. Eur J Nucl Med Mol Imaging 31: 325-329, 2004.

19. Pakos EE, Fotopoulos AD, Ioannidis JP. ${ }^{18} \mathrm{~F}$-FDG PET for evaluation of bone marrow infiltration in staging of lymphoma: a metaanalysis. J Nucl Med 46: 958-963, 2005.

(C) 2009 The Japanese Society of Internal Medicine http://www.naika.or.jp/imindex.html 